\title{
Electromagnetic field momentum from Feynman's paradox about space transformation mechanism
}

\author{
She Wang ${ }^{1,}$, Zhong Liu ${ }^{1}$ \\ ${ }^{1}$ Wuhan city vocational college, Wuhan City, Hubei province, China \\ a108988541@qq.com
}

\begin{abstract}
Keywords: Feynman's disc paradox; Dynamics; Electrodynamics; Conservation of momentum. Standing wave electromagnetic field; Electromagnetic induction. The momentum flow conversion; Mechanical and electrical interaction; MEMS; The spacecraft; All the electromagnetic propulsion.
\end{abstract}

\begin{abstract}
This article from introduce Feynman's disc paradox, the electromagnetic field energy and momentum conservation, For passive electromagnetic field's momentum flow and mechanical momentum of physical movement electrically charged particles transformation research, Passive electromagnetic field and electromagnetic field charged particles form always electromagnetic field To realize the electromagnetic momentum and electromagnetic momentum mechanical momentum transform mechanical momentum of electrically charged particles, Increase charged mechanical momentum of the system, Reduce electromagnetic momentum and flow of the electromagnetic momentum, To keep the electric system of mechanical momentum increment, System should be continuously added electromagnetic momentum and flow of the electromagnetic momentum, Continuous provide charged system of mechanical power, To solve the question of the motive forces of the deep sky long voyages, provide power theory foundation for development of large-scale universe.
\end{abstract}

\section{The dynamics of Feynman's disc paradox inference}

Feynman's disc paradox of reason is the law of conservation of momentum transfer of electromagnetic field.Show that under certain conditions, Electromagnetic angular momentum will into mechanical angular momentum, Into the whole process of the system total angular momentum remains the same, This shows that the space can happen electromagnetic induction electromagnetic field and charged system, Converts electromagnetic momentum or electromagnetic momentum flow mechanical momentum, Will also be part of the electromagnetic energy is converted to mechanical energy, This system, the change of the electromagnetic momentum, There is also a general Lorentz force $(\vec{f}=\rho \vec{E}+\vec{J} \times \vec{B})$ and the role of space electromagnetic flow momentum. The increase of system of mechanical momentum is equal to the space electromagnetic momentum and space electromagnetic momentum flow decreases, We concluded that electromagnetic field momentum space can be converted into mechanical momentum, Electromagnetic field as a form of the existence of matter, It has the energy, momentum and angular momentum, And you can with other forms of energy, momentum and angular momentum transformation, In the process of transformation should follow the law of conservation of their own, Feynman's disc is one of the important example involving the conversion, Feynman's proposed is a basic principle of electromagnetic field and field charged particles in the system of the general principle of conservation of angular momentum and momentum transfer.We can draw the following conclusion:

Feynman's disc system consists of power supply, long and thin solenoid

conductor, With the charge of balls;

Power supply Feynman's disc system of space electromagnetic field, Provide the electric field with the charge of the ball, the total electromagnetic field in the local space.

Feynman's disk system in space electromagnetic field change before and after the comply with the conservation of momentum and energy conservation.

Feynman's disk system mechanical momentum is derived from the momentum and momentum of the electromagnetic field flow of transformation. 
Power is the carrier of energy and momentum, Other forms of mechanical momentum and energy stored in the power carrier, power electromagnetic energy released by a certain form of circuit, Electromagnetic energy, electromagnetic momentum and electromagnetic angular momentum, total energy, momentum remains the same in the system.

Power stored in Feynman;s disc system part of momentum and energy into mechanical energy and momentum, Size is determined by the power supply.

Electromagnetic field only electromagnetic momentum and conservation of momentum in the infinite space mechanical momentum transformation, In local space, want to consider the effect of the electromagnetic momentum flow, By improving the circuit in the system, Will be more stable than Feynman disc system and constant mechanical momentum, The space electromagnetic momentum flow conversion system mechanical momentum of the generator.

In this paper, we study mainly reflects in:

Space electromagnetic field system was put forward momentum flow mechanism and the principle of electromagnetic momentum transfer into a mechanical momentum, Enriched the electromagnetic momentum and flow of the electromagnetic momentum and momentum and electromagnetic energy and mechanical energy conversion between mechanical theory.

\section{Space electromagnetic momentum flow conversion system mechanical momentum principle}

Above Feynman's disc system summary provides us a new way of thinking, Its difference with light pressure propulsion principle, Through the analysis of the electromagnetic momentum flow into mechanical momentum to drive the system movement, Rather than rely on the change of the electromagnetic momentum to transfer momentum, Similar to traditional motor principle, Through the magnetic field is the nature of the magnetic field force to conduction current, The transition of electromagnetic momentum flow system mechanical momentum, The force is called ampere force, It can do is work, Can also do positive work, Is the generator principle and principle of motor, Unlike traditional motor principle is the magnetic field is not the magnet object and excitation coil, etc.

Is provided by the magnetic field in electromagnetic waves, Thus eliminating the reaction of magnets and excitation coil, System object into a local space electromagnetic field and current system of one-on-one, Different from system composed of the motor and motor rotor and total electromagnetic field, In the system through a certain form of circuit and power supply conditions, The space electromagnetic mechanical momentum of the momentum flow into the system, To drive system movement.

\section{In this paper, the research content and discovery}

Standing wave electromagnetic field and there are interactions between a charged system.To force field of the electric system, A charged system after stress, Change its momentum, Standing wave at the same time the corresponding changes in the state of the electromagnetic field itself also.In fact, When the standing wave electromagnetic field coverage on the charged object, Charged particles in the system by the standing wave effect of electromagnetic field, The whole charged objects by electromagnetic force.After mechanical object, Its momentum change, Electromagnetic standing wave also changed its space motion state.In the process of this interaction, Standing wave electromagnetic flow momentum is transferred to a charged object, At the same time, the electromagnetic momentum of standing wave also corresponding change.Because standing wave electromagnetic field also and all other objects, have the momentum, And directly affect the occurrence of standing wave field of momentum and energy transfer to produce mechanical momentum and energy, The contribution of this paper research work mainly reflects in:This paper presents a standing wave electromagnetic field flow momentum transfer theory, It first from power transmission system to the specific circuit system, Electromagnetic momentum flow in the cavity body space, Electromagnetic induction and conduction current before and after, Through the general Lorentz force, The electromagnetic momentum flow mechanical momentum transfer, Enriched the 
electromagnetic and mechanical momentum, momentum between electromagnetic energy and mechanical energy conversion theory;Found the stable and effective mechanism of electromagnetic force, Innovation on electromagnetic flow momentum transfer to the theory of electromagnetic force, This wireless electromagnetic propulsion theory provides a foundation for further research and basis;Obtained can directly implement standing wave electromagnetic momentum, and energy to the mechanical momentum and mechanical energy conversion of the conclusion.

\section{Conclusion}

All electromagnetic induction phenomena available Lorentz magnetic force to make a comprehensive and accurate physical interpretation, Whether metal electrons cut magnetic field lines or magnetic linear cutting metal electrons, Its physical essence are charge by Lorentz magnetic force, From the electric field and charge density, magnetic field and the general Lorentz force density of current density, Demonstrated under a certain condition in electromagnetic wave between electromagnetic field and the current system can generate stable effective electromagnetic force, In a standing wave electromagnetic field flow momentum and energy is converted to mechanical momentum and mechanical energy.The electromagnetic wave is a kind of special material, But it is just like ordinary matter, Also has the momentum and energy, And can with ordinary matter, momentum and energy conversion, And meet the momentum and energy conservation.It is through electromagnetic we also in the process of the interaction between wave and current systems and electromagnetic current standing wave of momentum, energy and mechanical momentum of the current system and mechanical energy transformation, To get to know and obtain the electromagnetic interaction between standing wave and current system of mechanical and electrical.Dynamic principle of electromagnetic space are obtained, The results for the development of mechanics, electricity dynamics, MEMS technology, technical advance of the new theory of the guiding role of aeronautics and astronautics,

Can also be used in deep space motor power and massive cosmic development theoretical basis.

\section{References}

[1] J. Bouchard, B. Knoblich, H. Wicht. Will RF MEMS live up their promise?. In:Proceedings of the 36th European Microwave Conference, September 2006, $1076 \sim 1079$.

[2] S. Lucyszyn. Review of radio frequency microelectromechanical systems technology. In: IEE Proceedings - Science, Measurement and Technology, March 3, 2004, 151(2): 93 103.

[3] Kaihua Zhao, Josh seeks, electromagnetism. Beijing: higher education press. In April 2003. 106.

[4] A. Oida, H. Nakashima, J. Miyasaka, et al. Development of a new type of electric

off-road vehicle powered by microwaves transmitted through air. Journal of

Terramechanics, 2007, 44(5): 329 338.

[5] E. Shimane, S. Komatsu, K. Komurasaki, et al. A MAV flight using microwave power supply. In: 46th AIAA Aerospace Science Meeting and Exhibit, Reno, Nevada, January 7 10, 2008, AIAA 2008-1149.

[6] Zhong Liu. Research and discuss the electromagnetic momentum. Journal of

Reading and writing 2011, 38:55 56 In Chinese.

[7] DongLin Zu, electrodynamics Tsinghua university press, September 2006 the first edition 
[8] Yanjie Zhao, From a paradox to push the electromagnetic field of conservation of momentum theory Journal of shangqiu normal college in 2005

[9] Dan Xia, Tianlin Dong. Reflection and transmission of electromagnetic slow-waves by a uniformly moving dielectric slab. Chinese Science Bulletin, 2010, 55(34):3875 3879.

[10] Zhong Liu. The innovation of the electromagnetic propulsion theory research. Journal of The new curriculum 2013, 278:20 21 In Chinese.

[11] D. Xia, R. X. Yao, P. Li, et al. Measurement of transition frequency of composite transmission line model. In: Proceedings 2011 Asia-Pacific Power and Energy Engineering Conference (APPEEC 2011), Wuhan, China, March 25 28, 2011. 